\title{
FDA proposes new food labels
}

$\mathrm{T}$ he nutrition facts on packaged food labels in the United States may soon receive the most substantial update in their 20-year-history, announced the US Food and Drug Administration (FDA). The proposed label, open for public comment for 90 days, places more emphasis on the calorie count, updates out-of-date serving sizes and contains a new line for added sugars.

"Our guiding principle here is very simple: that you as a parent and a consumer should be able to walk into your local grocery store, pick up an item off the shelf, and be able to tell whether it's good for your family," First Lady Michelle Obama, a major force behind the proposed changes, stated in a press release. "So this is a big deal, and it's going to make a big difference for families all across this country."

The updates are based on new dietary recommendations, survey data and consensus reports. Nutrition information on packaged foods last received a major update in 2006, when the amount of trans fat was added.

Health advocates have long called for information about added sugars to be included on food labels, considering the effect that excessive sugar consumption has on obesity rates. Lumping added sugars together with naturally occurring sugars can send a mixed message and discourage people from consuming healthy foods such as fruits and low-fat dairy products, says Bill Jeffery, national coordinator for the Center for Science in the Public Interest.

"Adding a line for added sugar is definitely a step in the right direction," says Jeffery. "Food companies take advantage of people's ignorance about how much added sugar they consume."

Updates to serving sizes were necessary because they should be based on what people actually eat rather than what they "should" eat, according to the FDA. The portions typically consumed today are larger than in the 1990s. Now a serving size of ice cream, for example, will be one cup rather than half a cup. Again, this is a positive step, according to Jeffery.

"Providing nutrition information on unrealistically small serving sizes is doing a disservice," he says.

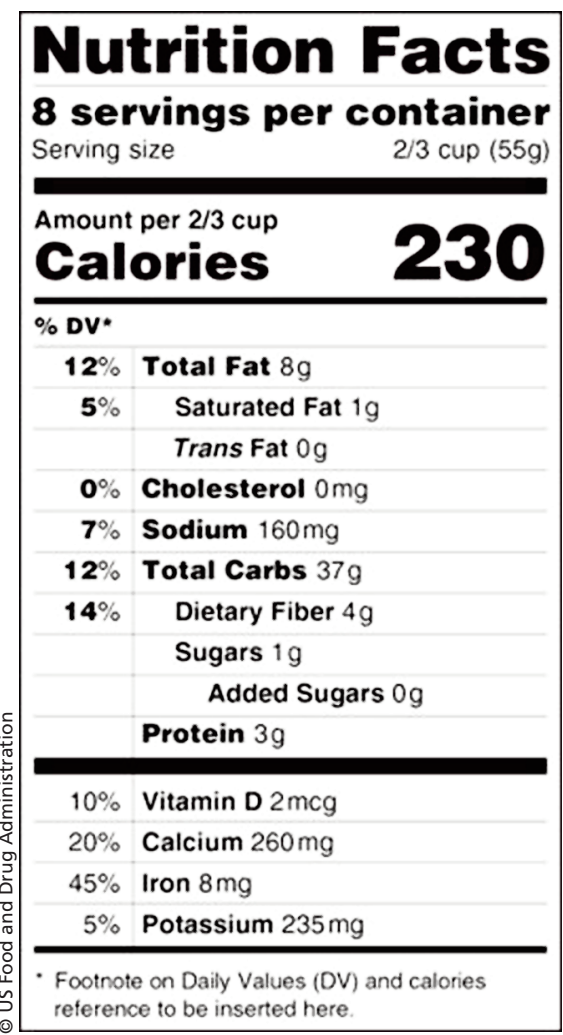

The proposed label places more emphasis on the calorie count, updates out-of-date serving sizes and includes added sugars.

At a glance, the most obvious change to the proposed label is the much larger font size for the calorie count. Anything that makes it easier for consumers to make healthy choices is a positive change, says Kate Comeau, a registered dietitian and spokesperson for Dietitians of Canada.

"Although we don't have a formal position on this, the Dietitians of Canada supports ways of making nutrition information easier to read, easier to find and easier to understand," says Comeau. "We see this as being in line with those goals."

Other changes to the proposed label include revisions to the percent daily values for some nutrients, such as sodium and dietary fibre, and the addition of potassium and vitamin $\mathrm{D}$, which contribute to bone health and lowering blood pressure. Declaring amounts of Vitamins $\mathrm{A}$ and $\mathrm{C}$ will not be required on the proposed label, though manufacturers can provide that information if they wish. - Roger Collier, CMAJ

CMAJ 2014. DOI:10.1503/cmaj.109-4748 